\title{
Cracking the Ubiquitin Code: The Ubiquitin Toolbox
}

\author{
Monique P.C. Mulder*, Katharina F. Witting and Huib Ovaa*
}

Oncode Institute and Department of Cell and Chemical Biology, Chemical Immunology, Leiden University Medical Centre, Leiden, the Netherlands.

${ }^{*}$ Correspondence: m.p.c.mulder@lumc.nl and h.ovaa@lumc.nl

https://doi.org/10.21775/cimb.037.001

\begin{abstract}
Ubiquitination, a post-translational modification, regulates a vast array of fundamental biological processes with dysregulation of the dedicated enzymes giving rise to pathologies such as cancer and neurodegenerative diseases. Assembly and its ensuing removal of this post-translational modification, determining a large variety of biological functions, is executed by a number of enzymes sequentially activating, conjugating, ligating, as well as deubiquitinating. Considering the vast impact of ubiquitination on regulating cellular homeostasis, understanding the function of these vast enzyme networks merits the development and innovation of tools. Thus, advances in synthetic strategies for generating ubiquitin, permitted the development of a plethora of ubiquitin assay reagents and numerous activity-based probes (ABPs) enable the study of enzymes involved in the complex system of ubiquitination. With ubiquitination playing such a pivotal role in the pathogenesis of a multitude of diseases, the identification of inhibitors for ubiquitin enzymes as well as the development of ABPs and high-throughput assay reagents is of utmost importance. Accordingly, this article will review the current state-of-the-art activity-based probes, reporter substrates, and other relevant tools based on $\mathrm{Ub}$ as a recognition element while highlighting the need of innovative technologies and unique concepts to study emerging facets of ubiquitin biology.
\end{abstract}

\section{Introduction}

One of the most versatile post-translational modifications is the attachment of the small protein ubiquitin ( $\mathrm{Ub})$ or its polymeric chains to target substrates. The attachment of the 76 amino acid long protein $\mathrm{Ub}$ to a nucleophilic functionality in the amino acid side chain of substrate proteins alters the fate of the modified protein, thereby regulating the vast majority of fundamental cellular processes such as DNA damage response (Muratani and Tansey, 2003), cell cycle progression (Kernan et al., 2018), transcription (Hicke, 2001), endocytosis (McCann et al., 2016), as well as apoptosis (Jackson and Durocher, 2013) and autophagy (Kwon and Ciechanover, 2017). Covalent attachment of $\mathrm{Ub}$ to its substrate proteins is orchestrated by the sequential action of three specialized enzyme classes - E1, E2, and E3 enzymes (Fig. 2.1A). However, the combination of E2 and E3 enzymes dictates what type of ubiquitin chain is formed and which substrate protein becomes ubiquitinated. To date, 2 human E1's, about 40 E2's and over 600 E3 enzymes are known. Adenylation of the $\mathrm{C}$-terminus of $\mathrm{Ub}$ at the expense of ATP yields a high-energy E1-Ub-thioester. Upon activation, $\mathrm{Ub}$ is transferred unto the active-site cysteine residue of the E2-enzyme, poising it for transfer unto the lysine residue of its substrates by the cooperation of an E3 enzyme. This final step in Ub-transfer through the E3 enzyme can occur via three main classes of E3 ligases: the homologous to the E6-AP- $\mathrm{C}$ terminus (HECT), the really interesting new gene (RING), 
A)

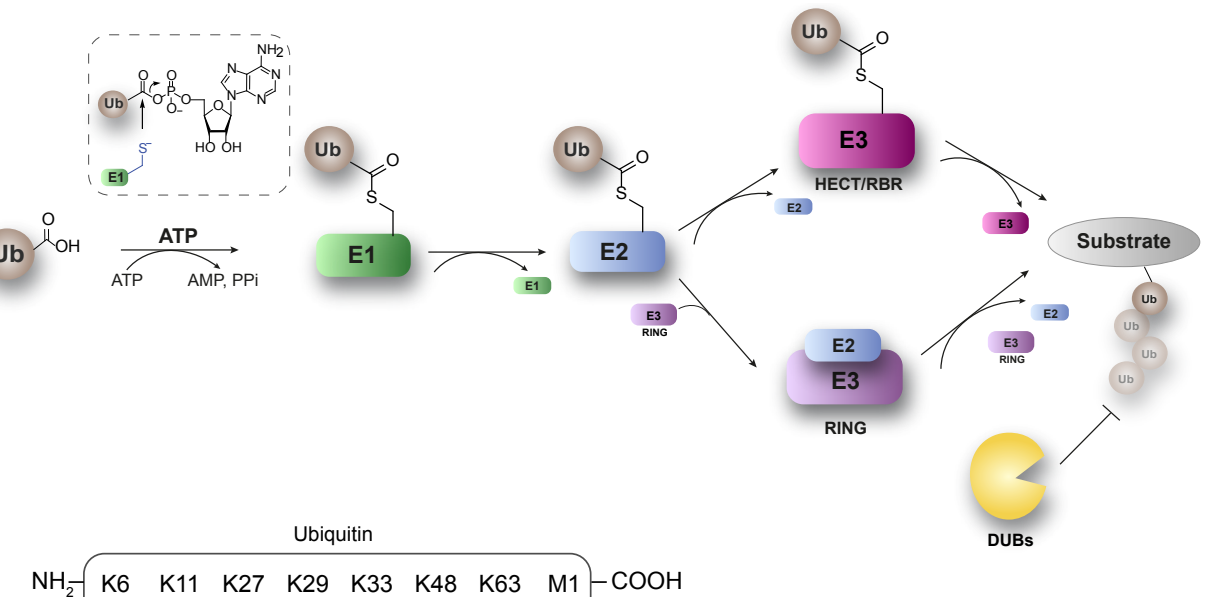

B)

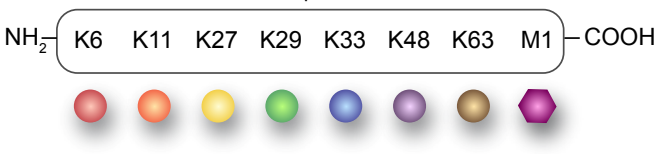

C) Homotypic

D)

Heterotypic

E) Hybrid
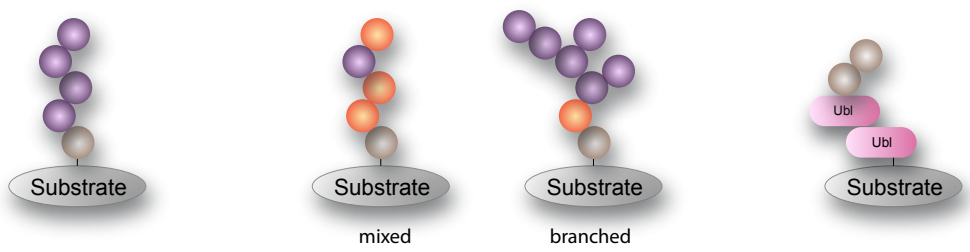

Figure 2.1 The complexity of ubiquitination. (A) The ubiquitination cascade, an orchestrated interplay of enzymes. (B) Self-modification of ubiquitin on one of its seven lysine residues results in a variety of different linkage types. Additionally, Ub can modify itself using the N-terminal methionine residue. (C) Increased complexity can be achieved by linking the Ub-modules in various manners leading to homotypic Ub-chains, in which the same type of Ub linkage is found or as (D) heterotypic linkages, which can either be mixed or branched. (E) Modification by a Ubl yielding hybrid chains.

and the RING-in-between-RING E3 (RBR) E3 enzymes (Vittal et al., 2015). In contrast to HECT E3 ligases, which utilize a direct transfer mechanism to relay activated ubiquitin to its substrate lysines, and RING E3s that employ an indirect scaffolding mechanism, RBR (RING-between-RING) ligases possess a trilateral domain architecture consisting of three zinc-binding domains - a RING1 domain flanked by an in-between-RING (IBR) domain, adjacent to a RING2 domain (Walden and Rittinger, 2018). On E2 Ub thioester recognition by RING1, it is transferred to the catalytic cysteine of RING2, which then facilitates transfer to the lysine of the substrate (Spratt et al., 2014; Walden and Rittinger, 2018).
Importantly, ubiquitination is a reversible process. The ubiquitination status of a protein can be regulated by removal or editing of ubiquitin chains, which is carried out by a group of approximately 100 deubiquitinating enzymes (DUBs) (Fig. 2.1A). Several categories of human DUBs have been identified to date; including the subfamilies of ubiquitin-specific proteases (USPs), ubiquitin C-terminal hydrolases (UCHs), Machado-Joseph disease proteases (MJD), ovarian tumour domain proteases (OTUs), motif interacting with Ubcontaining novel DUB family (MINDYs) and zinc finger with UFM1-specific peptidase domain protein (ZUFSPs) cleaving Ub linkages through a cysteine protease mechanism whereas JAB1/ 
MPN/MOV34 proteases (JAMMs) are zinc dependent metallo-proteases (Komander et al., 2009; Abdul Rehman et al., 2016; Hermanns et al., 2018). For some of these DUBs, linkage specificity has also been observed, further modulating the cellular response to ubiquitination (Komander and Rape, 2012; Harrigan et al., 2018). Intricate coordination of substrate ubiquitination by E3 ligases and DUBs is integral to maintain cellular homeostasis with deregulation leading to the onset and progression of numerous pathologies including cancer, neurodegenerative diseases, inflammatory, and infectious diseases arising from their deregulation (Scheffner and Kumar, 2014; Harrigan et al., 2018). This complex interplay is perhaps best exemplified by the ubiquitination of the tumour suppressor p53 by the E3 ligase MDM2 which is counterbalanced by USP7 deubiquitination thereby preventing proteasomal degradation but also regulating its expression levels (Nag et al., 2013).

To further modulate the biological consequence of Ubiquitination, $\mathrm{Ub}$ can undergo self-modification by forming isopeptide bonds between the $\mathrm{N}$-terminal methionine (Met1-linked ubiquitination) or any of the internal seven lysine (Lys6, Lys11, Lys27, Lys29, Lys33, Lys48, Lys63) $\varepsilon$-amines (Lys-linked ubiquitination) of one $\mathrm{Ub}$ molecule and the C-terminal carboxylic acid of another $\mathrm{Ub}$ molecule (Fig. 2.1B). In this manner, homotypic poly $\mathrm{Ub}$ chains of a single linkage type consisting of M1, K6, K11, K27, K29, K33, K48 or K63 can be formed (Fig. 2.1C), each having unique structural features creating distinct signalling events (Komander, 2009). While K48-Ub, one of the most abundant linkage type (Michel et al., 2017), destines substrates for proteasomal degradation, K33-linked Ubiquitin chains mediate protein trafficking (Yuan et al., 2014).

All of these linkages have been detected in cells and their abundance changes during specific cellular events, indicative of their various functions (Xu et al., 2009). In addition, heterotypic chains of multiple ubiquitin linkage types adopting mixed or branched topology can be formed (Fig. 2.1D), opening up an even more complex layer of posttranslational modification (Kim et al., 2007). The increased regulation of cellular processes especially by heterotypic ubiquitin chains is underscored by the observation that branched $\mathrm{K} 11 / \mathrm{K} 48$ Ubiquitin chains promote proteasomal degradation in vitro
(Meyer and Rape, 2014), while mixed K11/K63 linked Ubiquitin chains regulate the endocytic internalization of the major histocompatibility complex class 1 (MHC1) (Boname et al., 2010).

Additionally, $\mathrm{Ub}$ itself can be post translationally modified to further modulate the biological fate, most prominently by acetylation, phosphorylation, and more recently ribosylation (Yang et al., 2017). The consequences of such an additional modification is best exemplified by the phosphorylation of Ub by PINK1 resulting in Parkin recruitment and activation (Herhaus and Dikic, 2015). Furthermore, this additional layer of complexity can be expanded to include modification with Ubiquitinlike modifiers (Ubls) - a class of proteins that share high structural similarity and a common $\beta$-grasp fold with Ub such as SUMO, NEDD8 and ISG15 (Fig. 2.1E) (Kwon and Ciechanover, 2017). These UBL modifiers are attached to the target protein via their own dedicated E1, E2 and E3 enzymes and deconjugated with dedicated proteases.

Discovery of $\mathrm{Ub}$ and its role in proteasome mediated protein degradation was awarded with the Nobel Prize in Chemistry in 2004 (Giles, 2004). However, the complexity of the ubiquitination network and its cellular roles are far more diverse than just being a degradation signal. In the past years an enormous biochemical effort has been made in developing reagents and tools to study this complex enzyme cascade. Here, we will discuss the advances made in the chemical toolbox to study a broad range of biochemical and biological aspects of ubiquitin.

\section{Chemical approaches to ubiquitination}

In the past years, an enormous biochemical effort has been made in finding E2-E3 enzyme combinations that can give access to sufficient amounts of di- and polyubiquitin molecules representing all eight different homogenously linked ubiquitin types (Faggiano et al., 2016). In these efforts, people have been hampered by the lack of specific E2 and E3 enzymes to generate the so-called atypical (K6, K11, K27, K29, K33) chains. Only recently enzymatic approaches for making K6-, K11-, K29-, and K33-linked chains (Bremm et al., 2010; Hospenthal et al., 2013; Michel et al., 2015) were reported. Currently, only K27-linked ubiquitin 
remains enzymatically unattainable. On top of this some of the enzyme combinations reported are not linkage specific and further sample processing using DUBs (with their own specificity issues) is needed. Therefore, much effort has been put into making differentially linked ubiquitin derivatives or ubiquitinated proteins through semi-synthetic and synthetic strategies to circumvent traces of other linkages and assure homogenous preparation. Moreover, for study of the (de)ubiquitination network, modifying Ub derivatives with a specific handle to generate a particular Ub-based probe or enzyme substrate makes it even more challenging to prepare such a modified Ub conjugate enzymatically.

\section{Semi-synthetic strategies}

One of the most powerful semi-synthetic approaches for the production of large peptides and small proteins has been intein-based chemistry. This methodology relies on protein trans-splicing (PTS) which through a series of acyl shifts forms a thioester that can react with thiol or amine nucleophiles (Mootz, 2009). Expansion of the genetic code with unnatural amino acids (UAAs) has further aided the field of protein semi-synthesis and permitted the incorporation of unnatural amino acids facilitating the production of ubiquitin-based reagents (Trang et al., 2012; Wals and Ovaa, 2014; Rösner et al., 2015). While genetic code expansionbased methods are clearly useful, most do require certain expertise that can only be found in specialized labs and often require specific $E$. coli strains and tRNA pairs that might not be widely accessible. Another semi-synthetic strategy to generate fluorogenic ubiquitin and diubiquitin substrates exploits the E1-enzyme mediated C-terminal amidation reaction to equip the ubiquitin $\mathrm{C}$-terminus with several reactive groups (Wang et al., 2014).

\section{Synthetic strategies}

Although efforts to synthesize ubiquitin have been pioneered by Briand et al. (1989) and Ramage et al. (1994) in the late 1980s, the chemical synthesis of natively linked ubiquitinated peptide conjugates was first established by Muir and co-workers (Chatterjee et al., 2007). Their photo cleavable auxiliary (Aux) mediated ligation approach has paved the way for several chemical strategies for ubiquitination. Recently, two Aux mediated chemical ubiquitination methods have been reported. In the first approach Chatterjee and co-workers used a 2-aminooxyethanethiol Aux to mediate chemical ubiquitination (Weller et al., 2014). Their methodology enabled the preparation of the native isopeptide linkage by mild reductive removal of the Aux or alternatively, retention of the ligation Aux yielded protease-resistant non-native analogues of ubiquitinated peptides. Secondly, Liu and coworkers used the trifluoroacetic acid (TFA)-labile 1-(2,4-dimethoxyphenyl)-2-mercaptoethyl Aux to assist the synthesis of K27-linked di- and tri-Ub chains (Pan et al., 2016).

The native chemical ligation (NCL) reaction, an important extension of the chemical ligation field, is widely used to construct large poly peptides or proteins by reacting an $\mathrm{N}$-terminal cysteine residue to C-terminal thioester peptide followed by transthiolation and S-to-N-acyl migration giving an amide bond as final product (Dawson et al., 1994). This powerful technique, has been employed by Brik and co-workers and Ovaa and co-workers to synthesize Ub dimers of defined linkage by the incorporation of a $\delta$ - or $\gamma$-thiolysine moiety at a designated lysine residue to allow NCL with a thioester moiety, which had previously been introduced by Yang et al. (2009) (El Oualid et al., 2010; Kumar et al., 2010). Recently, this methodology was adapted to create Ub mutants containing both a thiolysineand a thioester entity, allowing polymerization under NCL conditions (van der Heden van Noort et al., 2017). The development of $\gamma$-thionorleucine (ThioNle) as handle for native chemical ligationdesulfurization has expanded the thiolated amino acid toolbox further and serves as a methionine substitute in NCL, making the N-terminal ubiquitination towards full synthetic linear M1 diubiquitin possible for the first time (Xin et al., 2018).

Liu and co-workers describe an alternative NCL strategy that does not require the use of the $\delta$ - or $\gamma$-thiolysine moieties. Here a premade isopeptidelinked Ub isomer, which has an N-terminal Cys and a C-terminal hydrazide, is the key building block to assemble atypical Ub chains in a modular fashion resulting in the synthesis of several linkage- and length-defined atypical Ub chains, including K27linked tetra-Ub and K11/K48-branched tri-, tetra-, penta-, and hexa-Ubs (Tang et al., 2017).

Only the introduction of an efficient linear Fmoc-based solid phase peptide synthesis (SPPS) 
of Ub unlocked the potential of the above described methodologies. The ubiquitin module can be synthesized with total linear synthesis, or from fragments. During the total linear Fmoc-based SPPS approach, the growing peptide chain is stabilized by the incorporation of special building blocks, that prevent the formation of aggregates as the $\mathrm{Ub}$ chain grows (El Oualid et al., 2010). These SPPS strategies have allowed for the site-specific installation of a wide variety of reactive groups, unnatural amino acids, fluorescent labels, or pull-down handles (Hameed et al., 2017). Recently, a microwave assisted SPPS methodology for ubiquitin was reported that avoids the use of aggregation breakers and allows synthesis of isoUb in just one day. Here a four segment three step ligation method is used to synthesize K33/K11 mixed triUb (Qu et al., 2018). Another study, exploits an intermolecular side reaction, observed while synthesizing $\mathrm{Ub}$ on a trityl resin, occurring between the $\mathrm{N}$-terminal amine of one $\mathrm{Ub}$ molecule and the activated $\mathrm{C}$-terminus of another $\mathrm{Ub}$ molecule to obtain natively M1-linked polymeric ubiquitin chains (van der Heden van Noort et al., 2018). The length of these M1-linked poly $\mathrm{Ub}$ chains (up to ten Ub-residues) is unprecedented in a single chemical reaction, giving easy access towards bona fide $\mathrm{M} 1$ poly $\mathrm{Ub}$ chains shown to be fully recognized by the enzymatic ubiquitination cascade, as exemplified by DUB (OTULIN) cleavage and E1 activation (Uba1). This research not only provides a platform for the development of novel tools based on polymeric Ub in the near future, but also highlights new insights important to consider in experimental design for the construction of large peptides (van der Heden van Noort et al., 2018).

Despite these technological advances, numerous aspects of $\mathrm{Ub}$ signalling are difficult to study with a native isopeptide bond. Since the proteolytic activity of DUBs degrades the poly-Ub chain, crystallization or pulldown experiments are rendered impossible. In order to study stable complexes between poly $\mathrm{Ub}$ chains and DUBs, catalytically inactive DUBs are typically used. Yet, this approach yields numerous drawbacks, especially in biological settings necessitating the use of proteolysis-resistant Ub-chains. Utilizing a variety of chemistries, a broad range of poly-Ubiquitin chains of all linkage types can be generated giving access to studying mechanistic aspects of DUB cleavage as well as elucidating the role of the Ub-chains in a cellular environment.

In the field of Ub-chemistry, examples of nonhydrolyzable $\mathrm{Ub}$ conjugates generating strategies include the oxime-based ligation (Shanmugham et al., 2010), Huisgen cycloaddition reaction between an alkyne and azide (Flierman et al., 2016) or thiol-ene chemistry leading to a forged thioether bridge (Valkevich et al., 2012). Of note is that the thus generated linkage between two following $\mathrm{Ub}$-modules is not the native isopeptide bond. Some of these unnatural linkages are generally accepted to be adequate amide-bond mimics and several examples show that poly Ub material containing this linkage is tolerated and advantageous in biological settings (Flierman et al., 2016; Zhang et al., 2017). It has however also been shown that slight modifications in this isopeptide linker region can have a dramatic effect on biological function (Haj-Yahya et al., 2012). Although synthetic strategies allow complete control over modifications, the experimental design needs to be carefully evaluated when using these reagents in biological settings to further the understanding of Ubiquitination.

Advantage of the chemical approaches described above over biochemical methods is the complete control over regioselectivity in the reaction and thus formation of only the desired (poly-) Ub chain. Another superiority is the potential ease of introducing modifications to the chain such as for instance incorporation of reactive groups on the C-terminal side converting the chains into an activity-based probe.

\section{Beyond ubiquitin - crosstalk with other post-translational modifications}

$\mathrm{Ub}$ itself can be post-translationally modified to further modulate the biological fate, and simple PTMs on $\mathrm{Ub}$ such as phosphorylation (Huguenin-Dezot et al., 2016) and acetylation (Ohtake et al., 2015) can be incorporated through semisynthetic approaches. However, more complex PTMs such as adenosine diphosphate ribose (ADPr), are more difficult to introduce. Interestingly, ADP-ribosylation of $\mathrm{Ub}$ (Arg42) is mediated by a family of effector proteins originating from Legionella pneumophila, the pathogen causing Legionnaires disease in an ATP-independent reaction to hijack the host 
cells $\mathrm{Ub}$ pool, preventing the processing of existing Ub chains by host DUBs, and use it to its own advantage. These SidE effectors are the first reported class of enzymes that are able to ubiquitinate target proteins independent of the normally employed enzymatic cascade of E1, E2, and E3 enzymes (Bhogaraju et al., 2016; Puvar et al., 2017). In a recent study, the design and synthesis of propargylated ADP-ribose building block is presented employing a copper-catalysed cycloaddition reaction in which an $\mathrm{Ub}$ azide (Arg42 replaced by azido-homoalanine) an analogue of Ub-ADPr, was prepared. Subsequently, this triazole-containing $\mathrm{Ub}-\mathrm{ADPr}$ was shown to be recognized in western blot and accepted by SdeA in an auto-ubiquitination assay, instigating a useful platform for the biological interrogation of Ub-ADPr biology (Liu et al., 2018).

Additionally, there is a growing evidence implying crosstalk between ubiquitin and ubiquitin-like (UbL) proteins, increasing the complexity and fine-tuning cellular responses further. Best studied is the crosstalk between ubiquitin and SUMO (Nie and Boddy, 2016), but ubiquitinated-NEDD8 chains and crosstalk between Ub and Nedd8 signalling pathways have also been reported (Leidecker et al., 2012; Singh et al., 2014), as well as the existence of ubiquitinated FAT10 (Buchsbaum et al., 2012) and ISGylated ubiquitin (Fan et al., 2015). To address these unmet needs on hybrid chains, (semi-)synthetic strategies for obtaining ubiquitinated Rubl, the yeast NEDD8 homologue (Singh et al., 2014) and SUMO-2-K63diUb hybrid chains (Bondalapati et al., 2017) have already been reported. Despite these advancements, synthetic strategies for obtaining full-length Ubl proteins have long been neglected. Only recently, efforts to devise synthetic strategies for $\mathrm{Ubl}$ proteins such as Nedd8 (Ekkebus et al., 2013), SUMO (Dobrotă et al., 2012; Wucherpfennig et al., 2014; Boll et al., 2015; Mulder et al., 2018) and Ufm1 (Ogunkoya et al., 2012; Witting et al., 2018) have been undertaken not only providing access to Ubl reagents allowing research on their respective enzymatic cascades, but also enabling future developments on hybrid chains enabling in depth studies on their crosstalk with ubiquitin.

\section{Visualizing ubiquitin in action - Ub reagents targeting DUBs and ligases}

Activity-based probes (ABPs) are powerful tools to study enzyme activities in vitro and in vivo and have been helpful for studying the activity of enzymes. They typically consist of three elements - a reactive group, a recognition element and a reporter tag and have been instrumental in not only identifying but also studying DUBs and more recently the conjugating and ligating enzymes of the $\mathrm{Ub}$ cascade (Hewings et al., 2017). Additionally, the introduction of a facile linear solid phase peptide synthesis method for ubiquitin, permitted the development of a plethora of ubiquitin assay reagents, such as fluorogenic assays, native and non-hydrolyzable ubiquitin-linkages, and even poly-ubiquitin chains thereby enabling the characterization of these enzymes.

\section{Taking a snapshot of DUB activity - ABPs targeting the deconjugation machinery}

While the first generation of ABPs targeting DUBs utilized Ubiquitin-aldehyde (UbaI) (Pickart and Rose, 1986) and Ub-nitrile (Ub-CN) (Lam et al., 1997), introduction of the vinyl-sulfone (VS) (Borodovsky et al, 2001) as a reactive group led to the development of irreversible DUB ABPs. Since then, a wide variety of electrophilic reactive groups (Borodovsky et al., 2002) have been introduced with the vinyl methyl ester (VME) (Borodovsky et al., 2002; Ovaa et al., 2004) and propargyl amides (PA) (Ekkebus et al., 2013) being the most widespread used ones (Fig. 2.2A). These ABPs furthered the discovery of novel DUBs, as is exemplified not only by the discovery of OTU family of DUBs (Borodovsky et al., 2002; Balakirev et al., 2003), numerous viral (Hewings et al., 2017) and bacterial DUBs (Pruneda et al., 2016), but also by the discovery of a novel bacterial protease class exhibiting both deubiquitinating and deneddylase activity (Grabe et al., 2016). In addition, they have been used in activity profiling, crystallization studies to study the interactions between the protease and $\mathrm{Ub}$ in detail as previously reviewed (van Tilburg et al., 2016), as well as inhibitor screening (Reverdy et al., 2012). However, these ABPs bind irreversibly to the active site of the DUB, rendering them 
A)

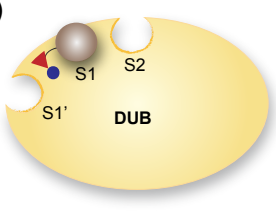

1st Generation

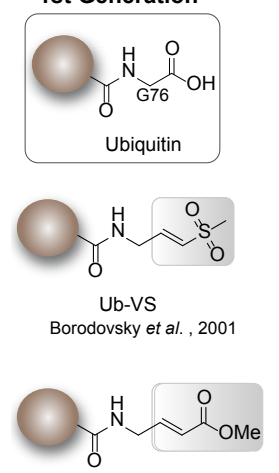

Ub-VME

Borodovsky et al., 2002

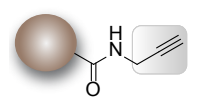

Ub-PA

Ekkebus et al., 2013
B)

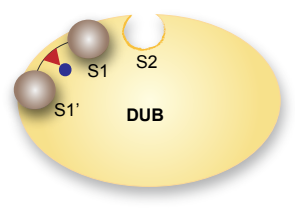

2nd Generation
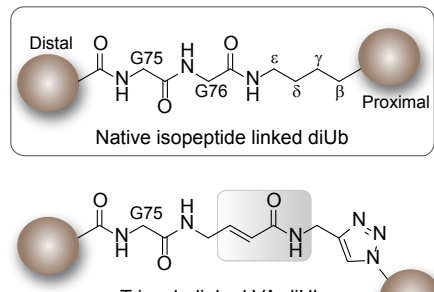

Triazole-linked VA-diUb (all seven linkages) McGouran et al., 2013
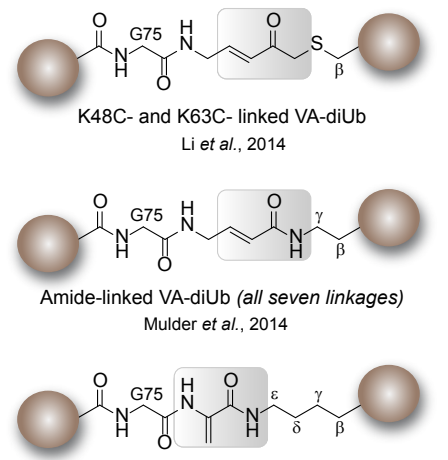

K48- and K63- linked Dha-diUb Haj-Yahya et al., 2014
C)

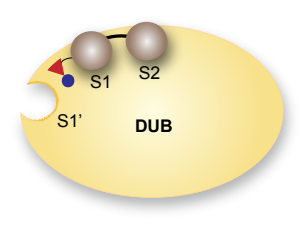

- catalytic site

4 warhead
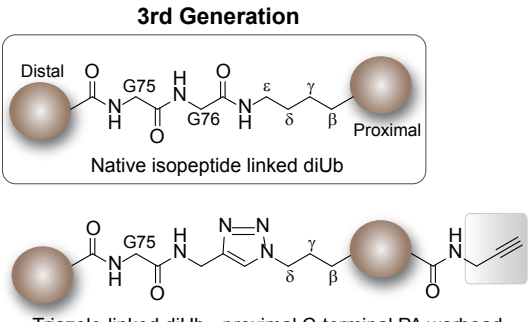

(all seven linkages) Flierman et al., 2016

Figure 2.2 Overview of activity-based probes to target DUB activity. (A) First generation DUB probes targeting S1 interactions. (B) Advanced DUB probes allowing S1 and S1' interactions. (C) Third generation DUB probes, enabling the covalent capture of DUBs preferentially targeting S1-S2 interactions.

inactive. In a recent study, a novel type of $\mathrm{ABP}$ containing a methyldisulfide warhead that captures DUBs reversibly, by means of active-site-specific disulphide exchange, allowing the release of an active enzyme was presented (de Jong et al., 2017). The significance of this probe lies in its ability to isolate active DUBs from their cellular environment retaining present cell-specific post-translational modifications that might regulate DUB activity. Although only proof of principal studies have been performed, this novel technology holds great promise for the future capture, release, and follow up investigations of native active cysteine DUBs in cellular contexts.
However, while activity-based probes have greatly increased our understanding of DUB reactivity and have enabled the discovery of new DUBs such as the OTU (Balakirev et al., 2003) and MINDY (MIU-containing novel DUB) classes (Abdul Rehman et al., 2016), these ABPs offer limited information on poly-Ubiquitin chain recognition and processing, since the existing diUbiquitin reagents contained isopeptide-linked Ubiquitin modules. While this characteristic allows the profiling of recombinant deubiquitinating enzymes towards their linkage specificity and kinetics (Mevissen et al., 2013), a major limitation is its incompatibility with the cellular environment 
which modulates DUB activity, thus necessitating innovative tools specifically addressing these questions. With the advent of synthetic strategies, a 2 nd generation of probes has emerged where, between two ubiquitin modules, a reactive group is positioned at the site of proteolytic action of the DUB allowing its covalent capture (Fig. 2.2B). An initial report by Iphofer et al. (2012) show a Michael acceptor linking the C-terminus of a distal $\mathrm{Ub}$ and short peptides representing K48 or K63 diUb. Later reports include the entire palette of Ub-chains allowing access to all seven lysine linked diubiquitin probes with a warhead in-between the distal and proximal ubiquitin module. Numerous research groups have independently reported ABPs utilizing a vinyl amide electrophilic trap between non-natively linked $\mathrm{Ub}$ moieties linked through a triazole, thiol ether (McGouran et al., 2013; Li et al., 2014) or an amide bond closely resembling the native isopeptide in both length as structure (Mulder et al., 2014). An alternative warhead is described by Haj-Yahya et al., here thiol elimination of $\mathrm{Ub}(\mathrm{G} 76 \mathrm{C})-\mathrm{Ub}$ results in dehydroalanine (Dha) as an electrophilic trap between two Ub modules (Haj-Yahya et al., 2014).

Although these covalent vinyl amide probes have allowed more detailed structural investigation of diubiquitin-specific DUB recognition (Mevissen et al., 2016), they do not allow investigation of additional Ubiquitin-binding sites, referred to as the S1' (proximal), S1 (middle), and S2 (distal) binding sites (Kulathu, 2016). To investigate the contribution of the Ubiquitin binding sites to polyubiquitin chain processing by DUBs, a third generation of probes (Fig. 2.2C) generated by click chemistry and C-terminally modified with propargyl (PA) were devised (Flierman et al., 2016). Utility of this reagent enabled the structural characterization of the K48 polyubiquitin cleaving mechanism of the SARS DUB PLpro, revealing that the S1-S1' binding mode of K48linked ubiquitin dictates the enzyme specificity for K48-Ubiquitin over ISG15, which binds only in the S1 site (Békés et al., 2016).

Despite the variety of di-ubiquitin-specific ABPs, designing effective tools to study the M1-linked chain type has posed a challenge primarily due to differences in chemistry imposed by the 'linear' peptide linkage. In attempts to create an linear diUb $\mathrm{ABP}$, the methionine 1 (M1) of the proximal $\mathrm{Ub}$ was replaced by the electrophilic dehydroalanine (Dha) residue. However, this probe was cleaved by OTULIN and USP2 rather than reacting covalently with the active site cysteine residues. A more recent design addressed this issue by replacing the Gly76 of the distal Ub by Dha (Weber et al., 2017). Although the $\mathrm{UbG}_{76} \mathrm{Dha}-\mathrm{Ub}$ probe showed high selectivity for OTULIN, it did not label other M1-cleaving DUBs, indicating that Gly76 of the distal $\mathrm{Ub}$ is essential for recognition and cleavage of linear diUb by other M1 cleaving DUBs. Interestingly, the first report on the fully synthetic preparation of linear diubiquitin reveals that the methionine to norleucine substitution of the proximal Ub affects the hydrolysis rate of DUBs towards the linear diUb chain (Xin et al., 2018). Assessment of DUB-mediated cleavage of the synthetic (NLE1linked) and expressed (M1-linked) linear diUb was assed using OTULIN, USP16 and USP21, known to specifically cleave the linear Ub linkage, demonstrated that synthetic NLE1-linked linear diUb was processed less efficiently than M1-linked linear diUb (Xin et al., 2018).

Collectively, these observations indicate a more profound role for methionine and Gly76 in the interaction between M1-linked diubiquitin and DUBs, complicating the way for the design of linear diUb-based activity-based probes and assay reagents. Furthermore, these ABPs together with the insights gained from both structural and biochemical studies underscore that the interaction dynamics of di-Ubiquitin chains are far more complex than previously assumed.

The numerous activity-based probes have furthered our mechanistic, kinetic, and biological understanding of DUBs as well as enabled the discovery of new DUB classes, yet these reagents do not target the JAMM/MPN and Machado-JacobDisease protein (MJD) metalloprotease DUBs. Developing such reagents akin to those for the other DUB families is urgently needed in order to dissect the role of these proteases in diseases.

While significant advances have been made in the development of a variety of activity-based probes and reagents for DUBs, similar tools are slowly emerging for the proteases specific for ubiquitinlike modifiers, such as for the de-SUMOylating (SENPs) (Mulder et al., 2018), de-NEDDylating (Ekkebus et al., 2013) and de-UFMylating enzymes (Witting et al., 2018). 


\section{Relaying ubiquitin to its substrate - ABPs targeting the ubiquitin conjugation machinery}

Whereas DUBs have been extensively profiled using $A B P s$, the Ub-conjugating and ligating enzymes have only recently become the focus of ABP development. The delay in developing suitable reagents to profile the E1-E2-E3 enzymes is largely due to the challenges attributed with targeting a sequential enzymatic cascade rather than a single enzyme.

While ABPs originally designed to specifically target DUBs, such as HA-Ub-VME and Ub-VS, display cross-reactivity with HECT E3 ligases, they are not designed for monitoring Ub-conjugating and ligating enzyme activity concurrently (Borodovsky et al., 2001; Love et al., 2009), necessitating the development of ABPs and reagents specifically devised for the Ub conjugation machinery.

At the apex of the ubiquitination cascade, the E1 enzyme activates the C-terminal carboxylate of ubiquitin in an ATP-dependent manner. In this initial step, the Ub-AMP adenylate is formed under the consumption of ATP and magnesium. Subsequently, the intermediate undergoes nucleophilic attack by the adjacent catalytic E1 active site cysteine resulting thioester bond and the simultaneous release of AMP (Olsen and Lima, 2013). Early efforts towards developing Ub-based probes targeting the E1-enzyme were pioneered by Lu et al. (2010), who used a C-terminal 5'-sulfonyladenosine modified Ub or Ubl. This design [Fig. 2.3A(I)] permitted the mechanistic study of the E1-catalysed adenylation and thioesterification by crosslinking it with the $\mathrm{Ub} / \mathrm{Ubl}$ probe. A major drawback of the semisynthetic approach taken by Lu et al. (2010) is the alteration of the $\mathrm{Ub} / \mathrm{Ubl}$ sequence. An and Statsyuk (2016) later published a method to efficiently generate the ABPs reported by $\mathrm{Lu}$ et al. (2010) while retaining the 'native' sequence, utilizing a native chemical ligation strategy followed by the conversion of cysteine to Dha, permitting the trapping of the 'tetrahedral E1-Ubl-AMP intermediate. Owing to the mechanism-based approach of these Ub/Ubl-AMP probes, it reacts directly with the E1-Ub/Ubl thioester intermediate resulting in the formation of the covalent Ub/Ubl-ABP1 conjugate structurally mimicking the Ub-AMP intermediate. Other advancements by Statsyuk and co-workers employed a mechanism-based approach [Fig. 2.3A(II)] using an AMP-derived compound (ABP1), which due to its structural resemblance of the $\mathrm{Ub} / \mathrm{Ubl}$-adenylate reacts with the $\mathrm{Ub} / \mathrm{Ubl}$ substrates rather than the respective E1 enzymes (An and Statsyuk, 2013). However, while this $A B P$ has the advantage of being cellpermeable, cross-reactivity issues limits its utility to monitoring ubiquitination of substrates in vitro. Together, these approaches all mimic the Ub-Ubladenylate intermediate restricting these $\mathrm{ABPs}$ to the $\mathrm{E} 1$, enabling them to be processed downstream the cascade towards E2 and HECT- and RBR-E3 enzymes.

The second step in the cascade involves transfer of the activated Ubiquitin from E1 to E2 via a thioester exchange reaction, a processes that can be trapped and studied using a $\mathrm{E} 2$ derived $\mathrm{ABP}$ [Fig. 2.3A(III)] (Stanley et al. 2015). Recombinant expression of an E2 and modification with a tosyl-substituted double activated ene-reagent (TDAE) forms an electron poor activated vinylsulfide that on juxta-positioning of the E1's cysteine is able to form a stable bis-thioether E1E2 complex (Stanley et al., 2015). To enable the study of enzymes downstream in the cascade a more advanced activity probe was designed (Fig. 2.3B) and generated in an analogues approach, coupling an azide-modified $\mathrm{Ub}$ to an alkynemodified tosyl-substituted doubly activated ene (TDAE) using click chemistry (Stanley et al., 2015; Pao et al., 2016). This design enabled the generation of stable E2-Ub conjugates, on reaction with a respective $\mathrm{E} 2$ enzyme, and subsequent recruitment of the RBR-E3 ligase Parkin whilst monitoring the transthiolation activity of this ligase (Pao et al., 2016). Of note is that in the TDAE derived probe the C-terminal RGG motif of $\mathrm{Ub}$ is replaced by the reactive TDAE element, which might limit the generality of such probes as it is implicated that R74 and the diGly motif can play an important role in recognition of the downstream enzymes (Zhao et al., 2012). In a later stage, Pao et al. (2018) include Arg74 in their TDAE-Ub probe and despite being the improper length, the $A B P s$ described are able to recruit not only HECT/RBR but also RING E3 ligases. Most notably, the authors discover a novel RING E3 ligase - MYCBP2 (or PRH1), which utilizes a unique cysteine relaying mechanism mediating the transfer of activated Ubiquitin onto the threonine and serine residues. This unexpected finding 
Mulder et al.

A) E1 probes

(I) Mimicking the tetrahedral intermediate of the E1-Ub-AMP complex.

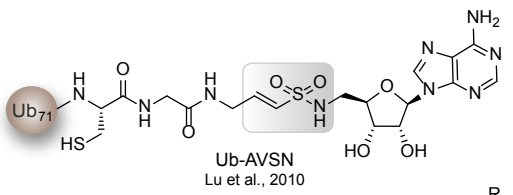

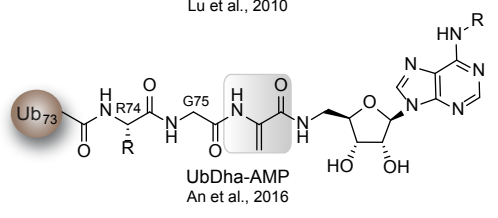

(II) Mechanism based probe consuming free Ub
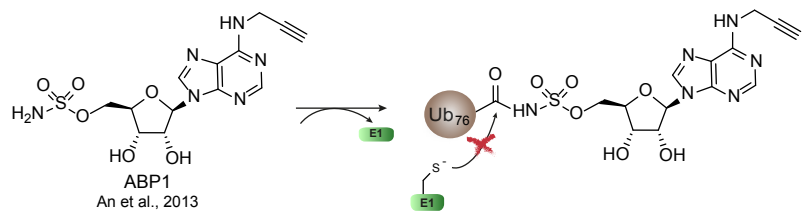

(III) ABPP of E1 enzyme tranthiolation activity

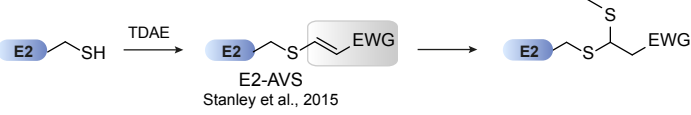

B) E2-E3 probes

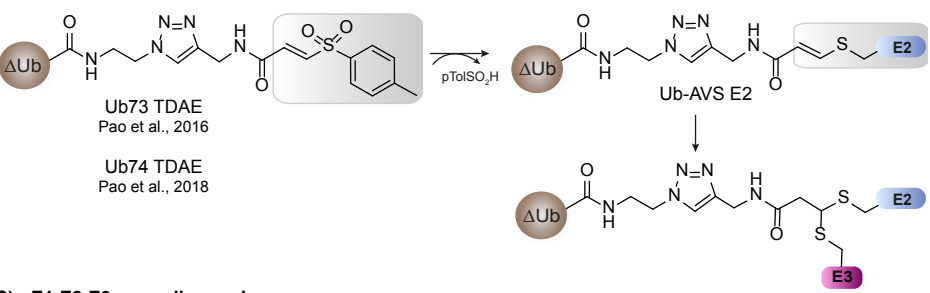

C) E1-E2-E3 cascading probe

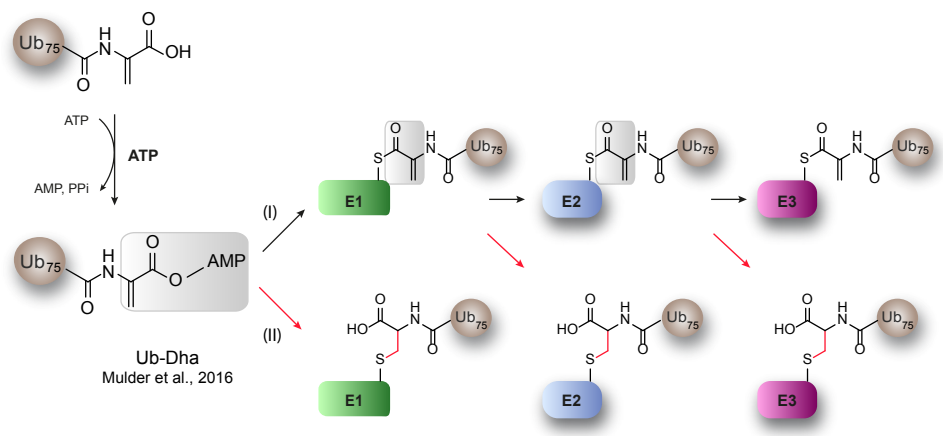

Figure 2.3 Current activity-based probes targeting the ubiquitin cascade. (A) Targeting the E1 enzyme in a mechanism-based manner by (I) mimicking the tetrahedral intermediate of the E1-Ub-AMP complex or (II) using an AMP-derived compound (ABP1) or (III) utilizing the E1-transthiolation activity. (B) Capturing Ub-E2-E3 interactions by a modular approach, where Ub-TDAE reacts with an E2 generating an ABP reactive towards HECT- and RBR- E3 ligases. (C) Cascading E1-E2-E3 ABP sequentially reacting with the E1, E2 and E3 enzymes by either forming (i) the thioester yielding the transferable Ub-probe or as (ii) a thioether, which allows irreversibly entrapment of the enzyme. 
exemplifies the utility and potential of ABPs and foreshadows the extent of future possibilities for these chemical tools (Pao et al., 2018).

In order to address the shortcomings of existing Ub-ABPs for studying multiple types of enzymes from the UPS simultaneously, Mulder et al. (2016) developed a mechanistically engaged $\mathrm{ABP}$ (Fig. 2.3C). Here the C-terminal Gly76 is replaced by Dha, thereby retaining a native carboxy terminus thus allowing it to be processed by the native $\mathrm{Ub}$ conjugation machinery in the same ATP-dependent manner with E1-Ub-based and E2-Ub-based probes transiently formed in situ allowing relay to the E2 and E3 enzymes. Most notably, at each transthiolation step, the probe also has the option of reacting covalently with the active site Cys. However, in contrast to native ubiquitin, this cascading probe is inert towards lysine residues in target proteins, making it applicable to chemo-proteomics approaches. Additionally, its ATP-dependant reactivity is advantageous for proteome-wide profiling experiments, as ATP-depletion permits facile background subtraction. Beyond its application for chemoproteomics, the utility of this unique cascading $\mathrm{ABP}$ has been showcased using living cells, where the effects of E1 enzyme inhibition on ubiquitination were visualized (Mulder et al., 2016). These experiments highlight the power of in-cell enzymology of the entire Ub cascade overcoming the limitation of labelling experiments in lysates, which are devoid of the organization and interaction of cellular structures.

The recent emergence of $\mathrm{E} 2-\mathrm{Ub}-\mathrm{ABPs}$ and the novel Ub-ABP Ub-Dha greatly expand the $\mathrm{Ub}$ toolbox and provide new ways to decipher the cellular functions and structural/biochemical properties of HECT ligases in specific cellular contexts as well as potentially in normal and disease state. However, of the three major classes of E3s, the current probes are only reactive towards HECT/RBR ligases, as these E3 ligases mechanistically rely on an active-site cysteine. RING E3s do not possess such an active site cysteine and merely serve as platforms to bring Ub charged E2's and substrates together, thereby making them unsuited for direct probing using ABPs.

\section{Assay reagents - real time monitoring of activity}

Measuring catalytic activity of (de)ubiquitinating enzymes is key not only to understand their biological function but also to inhibitor development efforts. In contrast to the probes described above these reagents lack a Michael acceptor element and thus do not form a covalent complex with their target enzymes, but instead rely on a fluorescent reporter tag allowing correlation of the enzymes native activity and/or specificity.

An important class of Ub based assay reagents are the fluorogenic assay reagents where a quenched fluorophore is conjugated via an amide bond at the C-terminal end of Ub. DUB activity and recognition will hydrolyse the amide bond at the $\mathrm{C}$-terminus of $\mathrm{Ub}$, releasing the fluorophore and simultaneously start to fluoresce. Hence the increase in fluorescence is a direct measure of DUB activity. One of the first fluorogenic reagents to measure the catalytic activity of DUBs is Ub aminomethyl coumarin (UbAMC) (Dang et al., 1998). Hassiepen et al. (2007) later report on a substituted rhodamine-110 (Rho110) scaffold with favourable fluorescent properties, making Ub-Rho110 a more preferred reagent in high throughput screening assays due to its non-overlapping spectrum with many small molecule inhibitors). In a similar set up, DUB mediated amino-luciferin release can be assayed in a bioluminescence approach using a luciferase assay, allowing the study of DUBs at lower concentrations (Orcutt et al., 2012). Another striking example illustrating the utility of fluorescent ubiquitin reagents are the non-hydrolyzable di-ubiquitin AMC reagents, which allow the monitoring of chain specific proteolysis mediated by S1-S2 interactions on the DUB. In analogy to the diUb-PRG covalent probes, these substrates allowed mechanistic dissection of DUB specificity and cleavage rate, exemplified by the finding that the $\mathrm{S} 2$ ubiquitin binding pocket of OTUD3 confers its preference for $\mathrm{K} 11 \mathrm{Ub}$-linkages as well as accelerating Ub hydrolysis (Flierman et al., 2016).

In all these cases, the reporters did not contain a native isopeptide bond at the side where the DUB would normally perform its proteolytic action, whereas the natural substrates for most 
DUBs would. Given that Ub-linkages govern a plethora of biological processes finetuning the cellular responses to a variety of stimuli, assessing the dynamics of Ubiquitin chain processing by DUBs is critical. Therefore, fluorescent polarization (FP) reagents were developed where $\mathrm{Ub}$ is conjugated via a native isopeptide linkage to a fluorophore carrying substrate derived peptide (Tirat et al., 2005; Geurink et al., 2012). Assays with these reagents are based on a change in fluorescence polarization on cleavage of the isopeptide bond between $\mathrm{Ub}$ and a fluorophore labelled peptide. While the unprocessed large Ub-FP reagents tumble slowly giving high fluorescence polarization, the processed small fluorophore containing peptide tumbles faster and hence the polarization of light decreases. The synthetic advancements enabled the generation of a palette of FP reagents as well as the generation of FP reagents based on UBLs like the three SUMO isoforms, NEDD8 and ISG15 (Geurink et al., 2012).

Another class of reagents are Fluorescent Resonance Energy Transfer (FRET)-based reagents that make use of a fluorophore and quenching moiety in close proximity of each other. On DUB proteolysis, the FRET signal decreases over time, which can be measured in a fluorescence spectrometer enabling the study of enzyme linkage specific kinetics in real time. Geurink et al. (2016) prepared all seven isopeptide-linked diUb FRET assay reagents by native chemical ligation using Rhodamine- $\mathrm{Ub}$ as the FRET-donor and TAMRA-Ub as the FRET-acceptor, permitting insights into the catalytic efficiency of vOTU. From the kinetic measurements it became apparent that the preference for K6-linked di-Ubiquitin chains over K48 chains resulted from an increased catalytic turnover rate $\mathrm{k}_{\mathrm{cat}}$ and not $\mathrm{Ub}$ binding $\left(\mathrm{K}_{\mathrm{M}}\right)$ (Geurink et al., 2016).

Using a similar technology, a high-throughput screening (HTS) assay for the E2 enzyme UBC13 was developed by combining a fluorochrome (Fl)conjugated ubiquitin (fluorescence acceptor) with terbium $(\mathrm{Tb}$ )-conjugated ubiquitin (fluorescence donor) in a TR-FRET assay, such that the assembly of mixed chains of Fl- and Tb-ubiquitin creates a robust TR-FRET signal. In this particular study, this reagent enabled the identification of $\mathrm{E} 2$ inhibitors (Madiraju et al., 2012).

While numerous reagents to assay the catalytic activity of DUBs have been reported, the development of reagents enabling the monitoring of Ubiquitin ligase activity has been lagging behind due to the complexity of these enzymes. An elegant attempt to generate reagents to efficiently monitor the transthiolation activity of HECT- and RBR-E3 ligases is the development of the 'Bypassing System' (ByS) by Park et al. (2015). This approach exploits a simple design - a Ub thioester mimic in the form of UbMES (mercaptoethanesulfonate), permitting the direct transthiolation of the catalytic cysteine of the E3 ligase while eliminating the need for the E1 and E2 enzymes as well as ATP. Further development of this concept led to the generation of a fluorescent $\mathrm{Ub}$ thioester permitting the detection of both transthiolation and ligation activities of HECT E3 ligases (Krist et al., 2016). Given the facile detection method and the requirement for only the E3 enzyme and UbFluor, this mechanismbased reagent is well suited for high throughput screens (HTS) for Ub ligase inhibitors (Foote et al., 2017).

\section{What does the future hold?}

Unravelling the complexity of the highly sophisticated ubiquitination system is aided greatly by the development of numerous ABPs and reagents reporting on the dynamics and structural mechanisms of (de)ubiquitinating enzymes involved. Given the intrinsic role of $\mathrm{Ub}$ in the pathogenesis of a variety of diseases, most notably cancer and neurodegenerative diseases, enzymes involved in this system are emerging drug targets. The utility of these activity-based probes and reagents has been showcased by the discovery and validation of a USP7 inhibitor utilizing both Ub-AMC in the initial high-throughput screen and later Ub-VS in the validation studies (Reverdy et al., 2012; Lamberto et al., 2017). Without a doubt the next generation of Ub based tools will help increase our knowledge, ultimately leading to new diagnostic tools or therapeutics making it to the clinic.

Although these recent advancements have helped gain insights into the functions of the engaged enzymes thereby facilitating more tailored solutions to interrogate their biology, it is becoming increasingly clear that these $\mathrm{ABPs}$ require innovation to address outstanding questions. The most pressing questions include dissecting DUB preference towards the Ub-linkage particularly 
of heterotypic and hybrid $\mathrm{Ub}$ chains; developing ABPs capable of capturing metalloprotease DUBs; advancing tools for specifically targeting distinct HECT and RBR-E3 ligases; and lastly, optimizing cell delivery methodologies for ABPs to enable incell enzymology.

\section{Customized tools - warranting study on a new complex layer of DUB recognition}

The advent of numerous $\mathrm{ABPs}$ and reagents for interrogating the different aspects of deubiquitinating enzymes, have enabled profound insights into the structural, biochemical and biological role of these 'erasers'. More recently, the generation of tools specifically designed for dissecting the proteolytic processing of ubiquitin chains by DUBs have revealed profound differences among these proteases in their specificity. Adding to this complexity, the discovery of heterotypic and hybrid Ubiquitin chains warrants the development of customized tools in order to understand the regulatory roles of DUBs in this context.

Given the recent insights that heterotypic Ubiquitin chains play a profound role in fine-tuning cellular responses (Xu et al., 2009), investigations into its biological and structural role need to be undertaken. To propel the study of their role, innovative $\mathrm{ABPs}$ recapitulating the structural and functional aspects of these mixed and branched Ubiquitin-chains need to be generated. Furthermore, the recent advances in synthetically obtaining Ubl proteins, permits the development of hybrid Ub/Ubl chains. Generation of such probes, especially for in-cell enzymology or proteomics context would be particularly conducive as the E3 ligases and DUBs regulating these heterotypic and hybrid Ub-chains are unknown (Xu et al., 2009). Furthermore, generating such complex linkages is a challenging feat as the E2/E3 enzymes generating these linkages in vitro are largely unknown and the known ones produce a mixture of linkage types that are difficult to separate by chromatography (Faggiano et al., 2016). Moreover, the modification of Ubiquitin or its Ubiquitin linkage by another PTM complicates the deciphering of the temporal order of events, which underlies the biological role of this modification. The urgent need for such ABPs and assay reagents is illustrated by the recently discovered MINDY DUBs, which preferentially cleave
K48 and K63 tetra-Ub linkages, raising the question whether they might display reactivity towards K48/K63 linkages (Xu et al., 2009; Ohtake and Tsuchiya, 2017). Since there are currently no ABPs recapitulating the mixed K48/K63 Ubiquitin linkage available, investigating this aspect is hampered.

Currently, the metalloprotease DUBs have been neglected in the development of ABPs and reagents partly due to the difficulty of designing these tools. Unlike other deubiquitinating enzymes, metalloprotease DUBs do not have an active-site cysteine, but instead hydrolyse the isopeptide bonds of ubiquitinated substrates with a water-coordinated zinc ion. Designing chemical probes with potent and specific zinc-ion chelating reactive groups is prerequisite to generating an innovative toolkit for metalloprotease DUBs. Generally, metalloproteases are typically expressed as an inactive form (zymogen) inhibited by additional proteins and require proteolytic processing before rendering the active enzyme (Saghatelian et al., 2004). This additional layer of regulation, however, introduces another layer of complexity that must be taken into account when designing such reagents (Saghatelian et al., 2004). Introducing such innovative chemical probes would propel the study of these understudied deubiquitinating enzymes and enable the development of therapeutics.

\section{The quest for E3 ligase inhibitors - challenges and opportunities}

Given that E3 ligases are involved in the pathogenesis of a variety of diseases, most notably cancer, neurodegenerative diseases such as Parkinson's, as well as numerous inflammatory diseases they are emerging drug targets (Goru et al., 2016; Uchida and Kitagawa, 2016). Although numerous assays, such as fluorogenic assays (Foote et al., 2017; Krist et al., 2017), FRET assays (Goldenberg et al., 2010), tandem ubiquitin-binding domains (Marblestone et al., 2012; Heap et al., 2017), bacterial or cellular two hybrid approaches (Levin-Kravets et al., 2016; Maculins et al., 2016), as well as biophysical methods (Regnström et al., 2013) have been reported, these approaches suffer from both low throughput, high number of false-positive or false-negative hits, and high costs. To overcome these shortcomings, a mass spectrometry-based assay using mono-ubiquitin to determine not only the E2/E3 enzyme activity facilitating highly sensitive and 
reproducible high-throughput inhibitor screening, was developed (De Cesare et al., 2018). Yet, one of the most challenging aspects to consider in such an undertaking is the lack of comprehensive prerequisite knowledge of the interacting E2-E3 enzyme pairs, which substantially modulate the biological outcome (De Cesare et al., 2018).

Despite this progress, the current ABPs targeting the ubiquitin conjugating cascade utilize either a modular approach (e.g. E2-Ub probe conjugates) or are mechanistic-based relying on the active-site cysteine (Mulder et al., 2016) or the ATP-binding pocket (An and Statsyuk, 2013, 2016) thereby being limited to indiscriminately detecting HECT- and RBR- E3-ligases. This limitation could potentially be overcome by designing ABPs featuring increased selectivity for HECT/RBR-E3 ligases by utilizing specific $\mathrm{Ub}$-variants generated by phage display (Zhang et al., 2016). Since some mechanistic aspects of $\mathrm{E} 3$ ligase-mediated catalysis is intrinsic to most E3 ligase probe designs, it excludes direct labelling of the scaffolding RING E3-ligases, which ironically comprise the vast majority of ligases that are pivotal in cancer development and progression (Wang et al., 2017). Yet, prerequisite for devising ABPs capable of selectively labelling RING E3 ligases is a priori knowledge of the specific interfaces between E2 and RING-E3 enzyme amenable to protein-protein interaction disruption.

\section{Probing ubiquitination in living cells}

Most ABP profiling experiments are performed using either recombinant enzymes or cell lysates, yet this does not recapitulate the activity of the enzymes in a cellular context. Since lysing cells results in disruption of the cellular compartmentalization as well as in dilution of the enzymes which might affect enzyme reactivity, delivery of DUB and ubiquitin ligase ABPs into intact cells is of critical importance. However, to achieve this, several methods including electroporation (Mulder et al., 2016) or the use of cell-penetrating peptides attached to the Ub-ABP (Gui et al., 2018; Hameed et al., 2018) have been reported. Additionally, the introduction of ABPs into living cells permit the visualization and in-cell enzymology of the ubiquitin cascade enzymes in a spatial and temporal context. The critical need for an intact cellular environment for proper enzymatic function of Ubiquitin enzymes arises from the interaction with protein complexes as well as their substrates, but also the intrinsic regulation by cellular signalling events such as phosphorylation (Sowa et al., 2009; Heideker and Wertz, 2015). The significance of additional posttranslational modification, e.g. phosphorylation, of DUBs to enhance their proteolytic activity is highlighted by the necessity of serine phosphorylation of OTUD5/DUBA (Huang et al., 2012). Furthermore, cross-regulation of DUBs with E2 enzymes (Wiener et al., 2012) and E3-ligases (Heideker and Wertz, 2015) underscore the significance of studying the ubiquitin cascade in living cells. One notable example of aforementioned interactions is the well characterized deubiquitinating enzyme USP7, which binds to the E3 ligase MDM2 and its substrate tumour suppressor p53 through its TRAF-domain (Sheng et al., 2006). Considering the significance of an functional cellular environment for the enzymatic function of the ubiquitin enzymes, their biochemical study should be conducted in living cells thus meriting ABPs compatible with in-cell enzymology. Another facet necessitating in-cell enzymology using ABPs is the application in proteomics to access not only the functional consequence of these interactions, particularly in the context of pharmacological inhibition (De Cesare et al., 2018).

\section{Conclusion}

Since the first ABP targeting DUBs, the field has brought forth an assortment of tools for interrogating a wide scope of biochemical and structural questions. The ensuing course of development illustrates how the development of activity-based probes and assay reagents for DUBs led to the discovery of new DUBs subsequently spawning the innovation of specialized reagents. While a variety of tools are reported for DUBs, the complexity of sequentially targeting an enzymatic cascade hampered the development of analogous advancements for the ubiquitin activating, conjugating, and ligating enzymes. Although the first ABPs targeting the ubiquitin activating enzyme have been reported almost a decade ago, reagents for the downstream enzymes are now slowly starting to emerge. One $\mathrm{ABP}$ that stands out is UbDha, which has the unique capability of being sequentially transferred through the ubiquitin cascade in a manner reminiscent to native Ubiquitin. Conclusively, the current 
platform of reagents and ABPs have the potential to accelerate drug discovery efforts targeting all aspects of the ubiquitin cascade. Yet, the frontier of Ubiquitin activity-based probe and reagent development lies in the introduction of innovative technologies and unique concepts enabling the dissection of many enigmatic aspects of ubiquitination as well as accessing enzymes previously not targeted by conventional $\mathrm{ABP}$ designs.

\section{References}

Abdul Rehman, S.A., Kristariyanto, Y.A., Choi, S.Y., Nkosi, P.J., Weidlich, S., Labib, K., Hofmann, K., and Kulathu, Y. (2016). MINDY-1 is a member of an evolutionarily conserved and structurally distinct new family of deubiquitinating enzymes. Mol. Cell 63, 146-155. https://doi.org/10.1016/j.molcel.2016.05.009.

An, H., and Statsyuk, A.V. (2013). Development of activitybased probes for ubiquitin and ubiquitin-like protein signaling pathways. J. Am. Chem. Soc. 135, 16948 16962. https://doi.org/10.1021/ja4099643.

An, H., and Statsyuk, A.V. (2016). Facile synthesis of covalent probes to capture enzymatic intermediates during E1 enzyme catalysis. Chem. Commun. 52, 2477-2480. https://doi.org/10.1039/c5cc08592f.

Balakirev, M.Y., Tcherniuk, S.O., Jaquinod, M., and Chroboczek, J. (2003). Otubains: a new family of cysteine proteases in the ubiquitin pathway. ЕMBO Rep. 4, 517-522. https://doi.org/10.1038/sj.embor. embor824.

Békés, M., van der Heden van Noort, G.J., Ekkebus, R., Ovaa, H., Huang, T.T., and Lima, C.D. (2016). Recognition of Lys48-linked di-ubiquitin and deubiquitinating activities of the SARS coronavirus papain-like protease. Mol. Cell 62, 572-585. https://doi.org/10.1016/j. molcel.2016.04.016.

Bhogaraju, S., Kalayil, S., Liu, Y., Bonn, F., Colby, T., Matic, I., and Dikic, I. (2016). Phosphoribosylation of ubiquitin promotes serine ubiquitination and impairs conventional ubiquitination. Cell 167, 1636-1649.e13.

Boll, E., Drobecq, H., Ollivier, N., Blanpain, A., Raibaut, L., Desmet, R., Vicogne, J., and Melnyk, O. (2015). Onepot chemical synthesis of small ubiquitin-like modifier protein-peptide conjugates using bis(2-sulfanylethyl) amido peptide latent thioester surrogates. Nat. Protoc. 10, 269-292. https://doi.org/10.1038/nprot.2015.013.

Boname, J.M., Thomas, M., Stagg, H.R., Xu, P., Peng, J., and Lehner, P.J. (2010). Efficient internalization of MHC I requires lysine- 11 and lysine- 63 mixed linkage polyubiquitin chains. Traffic 11, 210-220. https://doi. org/10.1111/j.1600-0854.2009.01011.x.

Bondalapati, S., Eid, E., Mali, S.M., Wolberger, C., and Brik, A. (2017). Total chemical synthesis of SUMO-2-Lys63linked diubiquitin hybrid chains assisted by removable solubilizing tags. Chem. Sci. 8, 4027-4034. https://doi. org/10.1039/c7sc00488e.

Borodovsky, A., Kessler, B.M., Casagrande, R., Overkleeft, H.S., Wilkinson, K.D., and Ploegh, H.L. (2001). A novel active site-directed probe specific for deubiquitylating enzymes reveals proteasome association of USP14.
EMBO J. 20, 5187-5196. https://doi.org/10.1093/ emboj/20.18.5187.

Borodovsky, A., Ovaa, H., Kolli, N., Gan-Erdene, T., Wilkinson, K.D., Ploegh, H.L., and Kessler, B.M. (2002). Chemistry-based functional proteomics reveals novel members of the deubiquitinating enzyme family. Chem. Biol. 9, 1149-1159.

Bremm, A., Freund, S.M., and Komander, D. (2010). Lys11linked ubiquitin chains adopt compact conformations and are preferentially hydrolyzed by the deubiquitinase Cezanne. Nat. Struct. Mol. Biol. 17, 939-947. https:// doi.org/10.1038/nsmb.1873.

Briand, J.P., Van Dorsselaer, A., Raboy, B., and Muller, S. (1989). Total chemical synthesis of ubiquitin using BOP reagent: biochemical and immunochemical properties of the purified synthetic product. Pept. Res. 2, 381-388.

Buchsbaum, S., Bercovich, B., and Ciechanover, A. (2012). FAT10 is a proteasomal degradation signal that is itself regulated by ubiquitination. Mol. Biol. Cell 23, 225-232. https://doi.org/10.1091/mbc.E11-07-0609.

Chatterjee, C., McGinty, R.K., Pellois, J.P., and Muir, T.W. (2007). Auxiliary-mediated site-specific peptide ubiquitylation. Angew. Chem. Int. Ed. Engl. 46, 28142818. https://doi.org/10.1002/anie.200605155.

Dang, L.C., Melandri, F.D., and Stein, R.L. (1998). Kinetic and mechanistic studies on the hydrolysis of ubiquitin C-terminal 7-amido-4-methylcoumarin by deubiquitinating enzymes. Biochemistry 37, 18681879. https://doi.org/10.1021/bi9723360.

Dawson, P.E., Muir, T.W., Clark-Lewis, I., and Kent, S.B. (1994). Synthesis of proteins by native chemical ligation. Science 266, 776-779.

De Cesare, V., Johnson, C., Barlow, V., Hastie, J., Knebel, A., and Trost, M. (2018). The MALDI-TOF E2/E3 ligase assay as universal tool for drug discovery in the ubiquitin pathway. Cell Chem. Biol. 25, 1117-1127.e4.

de Jong, A., Witting, K., Kooij, R., Flierman, D., and Ovaa, H. (2017). Release of enzymatically active deubiquitinating enzymes upon reversible capture by disulfide ubiquitin reagents. Angew. Chem. Int. Ed. Engl. 56, 12967-12970. https://doi.org/10.1002/anie.201706738.

Dobrotă, C., Fasci, D., Hădade, N.D., Roiban, G.D., Pop, C., Meier, V.M., Dumitru, I., Matache, M., Salvesen, G.S., and Funeriu, D.P. (2012). Glycine fluoromethylketones as SENP-specific activity based probes. Chembiochem 13, 80-84. https://doi.org/10.1002/cbic.201100645.

Ekkebus, R., van Kasteren, S.I., Kulathu, Y., Scholten, A., Berlin, I., Geurink, P.P., de Jong, A., Goerdayal, S., Neefjes, J., Heck, A.J., et al. (2013). On terminal alkynes that can react with active-site cysteine nucleophiles in proteases. J. Am. Chem. Soc. 135, 2867-2870. https:// doi.org/10.1021/ja309802n.

El Oualid, F., Merkx, R., Ekkebus, R., Hameed, D.S., Smit, J.J., de Jong, A., Hilkmann, H., Sixma, T.K., and Ovaa, H. (2010). Chemical synthesis of ubiquitin, ubiquitinbased probes, and diubiquitin. Angew. Chem. Int. Ed. Engl. 49, 10149-10153. https://doi.org/10.1002/ anie.201005995.

Faggiano, S., Alfano, C., and Pastore, A. (2016). The missing links to link ubiquitin: Methods for the enzymatic production of polyubiquitin chains. Anal. Biochem. 492, 82-90. https://doi.org/10.1016/j.ab.2015.09.013. 
Fan, J.B., Arimoto, K., Motamedchaboki, K., Yan, M., Wolf, D.A., and Zhang, D.E. (2015). Identification and characterization of a novel ISG15-ubiquitin mixed chain and its role in regulating protein homeostasis. Sci. Rep. 5, 12704. https://doi.org/10.1038/srep12704.

Flierman, D., van der Heden van Noort, G.J., Ekkebus, R., Geurink, P.P., Mevissen, T.E., Hospenthal, M.K., Komander, D., and Ovaa, H. (2016). Non-hydrolyzable diubiquitin probes reveal linkage-specific reactivity of deubiquitylating enzymes mediated by $\mathrm{S} 2$ pockets. Cell Chem. Biol. 23, 472-482. https://doi.org/10.1016/j. chembiol.2016.03.009.

Foote, P.K., Krist, D.T., and Statsyuk, A.V. (2017). Highthroughput screening of HECT E3 ubiquitin ligases using UbFluor. Curr. Protoc. Chem. Biol. 9, 174-195. https://doi.org/10.1002/cpch.24.

Geurink, P.P., El Oualid, F., Jonker, A., Hameed, D.S., and Ovaa, H. (2012). A general chemical ligation approach towards isopeptide-linked ubiquitin and ubiquitin-like assay reagents. Chembiochem 13, 293-297. https://doi. org/10.1002/cbic.201100706.

Geurink, P.P., van Tol, B.D., van Dalen, D., Brundel, P.J., Mevissen, T.E., Pruneda, J.N., Elliott, P.R., van Tilburg, G.B., Komander, D., and Ovaa, H. (2016). Development of diubiquitin-based FRET probes to quantify ubiquitin linkage specificity of deubiquitinating enzymes. Chembiochem 17, 816-820. https://doi.org/10.1002/ cbic. 201600017.

Giles, J. (2004). Chemistry Nobel for trio who revealed molecular death-tag. Nature 431, 729.

Goldenberg, S.J., Marblestone, J.G., Mattern, M.R., and Nicholson, B. (2010). Strategies for the identification of ubiquitin ligase inhibitors. Biochem Soc Trans 38, 132-136. https://doi.org/10.1042/BST0380132.

Goru, S.K., Pandey, A., and Gaikwad, A.B. (2016). E3 ubiquitin ligases as novel targets for inflammatory diseases. Pharmacol. Res. 106, 1-9.

Grabe, G.J., Zhang, Y., Przydacz, M., Rolhion, N., Yang, Y., Pruneda, J.N., Komander, D., Holden, D.W., and Hare, S.A. (2016). The Salmonella effector SpvD is a cysteine hydrolase with a serovar-specific polymorphism influencing catalytic activity, suppression of immune responses, and bacterial virulence. J. Biol. Chem. 291, 25853-25863.

Gui, W., Ott, C.A., Yang, K., Chung, J.S., Shen, S., and Zhuang, Z. (2018). Cell-permeable activity-based ubiquitin probes enable intracellular profiling of human deubiquitinases. J. Am. Chem. Soc. 140, 12424-12433. https://doi.org/10.1021/jacs.8b05147.

Haj-Yahya, M., Eltarteer, N., Ohayon, S., Shema, E., Kotler, E., Oren, M., and Brik, A. (2012). N-methylation of isopeptide bond as a strategy to resist deubiquitinases. Angew. Chem. Int. Ed. Engl. 51, 11535-11539. https:// doi.org/10.1002/anie.201205771.

Haj-Yahya, N., Hemantha, H.P., Meledin, R., Bondalapati, S., Seenaiah, M., and Brik, A. (2014). Dehydroalaninebased diubiquitin activity probes. Org. Lett. 16, 540-543. https://doi.org/10.1021/ol403416w.

Hameed, D.S., Sapmaz, A., and Ovaa, H. (2017). How chemical synthesis of ubiquitin conjugates helps to understand ubiquitin signal transduction. Bioconjug. Chem. 28, 805-815. https://doi.org/10.1021/acs. bioconjchem.6b00140.
Hameed, D.S., Sapmaz, A., Gjonaj, L., Merkx, R., and Ovaa, H. (2018). Enhanced Delivery of Synthetic Labelled Ubiquitin into Live Cells by Using Next-Generation Ub-TAT Conjugates. Chembiochem. 19, 2553-2557.

Harrigan, J.A., Jacq, X., Martin, N.M., and Jackson, S.P. (2018). Deubiquitylating enzymes and drug discovery: emerging opportunities. Nat. Rev. Drug Discov. 17, 57-78. https://doi.org/10.1038/nrd.2017.152.

Hassiepen, U., Eidhoff, U., Meder, G., Bulber, J.F., Hein, A., Bodendorf, U., Lorthiois, E., and Martoglio, B. (2007). A sensitive fluorescence intensity assay for deubiquitinating proteases using ubiquitin-rhodamine110-glycine as substrate. Anal. Biochem. 371, 201-207.

Heap, R.E., Gant, M.S., Lamoliatte, F., Peltier, J., and Trost, M. (2017). Mass spectrometry techniques for studying the ubiquitin system. Biochem. Soc. Trans. 45, 11371148. https://doi.org/10.1042/BST20170091.

Heideker, J., and Wertz, I.E. (2015). DUBs, the regulation of cell identity and disease. Biochem. J. 467, 191.

Herhaus, L., and Dikic, I. (2015). Expanding the ubiquitin code through post-translational modification. EMBO Rep. 16, 1071-1083. https://doi.org/10.15252/ embr.201540891.

Hermanns, T., Pichlo, C., Woiwode, I., Klopffleisch, K., Witting, K.F., Ovaa, H., Baumann, U., and Hofmann, K. (2018). A family of unconventional deubiquitinases with modular chain specificity determinants. Nat. Commun. 9, 799. https://doi.org/10.1038/s41467-018-03148-5.

Hewings, D.S., Flygare, J.A., Bogyo, M., and Wertz, I.E. (2017). Activity-based probes for the ubiquitin conjugation-deconjugation machinery: new chemistries, new tools, and new insights. FEBS J. 284, 1555-1576. https://doi.org/10.1111/febs.14039.

Hicke, L. (2001). Protein regulation by monoubiquitin. Nat. Rev. Mol. Cell Biol. 2, 195-201. https://doi. org $/ 10.1038 / 35056583$.

Hospenthal, M.K., Freund, S.M., and Komander, D. (2013). Assembly, analysis and architecture of atypical ubiquitin chains. Nat. Struct. Mol. Biol. 20, 555-565. https://doi. org/10.1038/nsmb.2547.

Huang, O.W., Ma, X., Yin, J., Flinders, J., Maurer, T., Kayagaki, N., Phung, Q. Bosanac, I., Arnott, D., Dixit, V.M., et al. (2012). Phosphorylation-dependent activity of the deubiquitinase DUBA. Nat. Struct. Mol. Biol. 19, 171-175. https://doi.org/10.1038/nsmb.2206.

Huguenin-Dezot, N., De Cesare, V., Peltier, J., Knebel, A., Kristaryianto, Y.A., Rogerson, D.T., Kulathu, Y., Trost, M., and Chin, J.W. (2016). Synthesis of isomeric phosphoubiquitin chains reveals that phosphorylation controls deubiquitinase activity and specificity. Cell Rep. 16, 1180-1193.

Iphofer, A., Kummer, A., Nimtz, M., Ritter, A., Arnold, T., Frank, R., van den Heuvel, J., Kessler, B.M., Jansch, L., and Franke, R. (2012). Profiling ubiquitin linkage specificities of deubiquitinating enzymes with branched ubiquitin isopeptide probes. Chembiochem. 13, 14161420.

Jackson, S.P., and Durocher, D. (2013). Regulation of DNA damage responses by ubiquitin and SUMO. Mol. Cell 49, 795-807. https://doi.org/10.1016/j. molcel.2013.01.017.

Kernan, J., Bonacci, T., and Emanuele, M.J. (2018). Who guards the guardian? Mechanisms that restrain APC/C 
during the cell cycle. Biochim. Biophys. Acta Mol. Cell Res. 1865, 1924-1933.

Kim, H.T., Kim, K.P., Lledias, F., Kisselev, A.F., Scaglione, K.M., Skowyra, D., Gygi, S.P., and Goldberg, A.L. (2007). Certain pairs of ubiquitin-conjugating enzymes (E2s) and ubiquitin-protein ligases (E3s) synthesize nondegradable forked ubiquitin chains containing all possible isopeptide linkages. J. Biol. Chem. 282, 17375-17386.

Komander, D. (2009). The emerging complexity of protein ubiquitination. Biochem Soc Trans 37, 937953.10.1042/BST0370937.

Komander, D., and Rape, M. (2012). The ubiquitin code. Annu. Rev. Biochem. 81, 203-229. https://doi. org/10.1146/annurev-biochem-060310-170328.

Komander, D., Clague, M.J., and Urbé, S. (2009). Breaking the chains: structure and function of the deubiquitinases. Nat. Rev. Mol. Cell Biol. 10, 550-563. https://doi. org/10.1038/nrm2731.

Krist, D.T., Park, S., Boneh, G.H., Rice, S.E., and Statsyuk, A.V. (2016). UbFluor: a mechanism-based probe for HECT E3 ligases. Chem. Sci. 7, 5587-5595. https:// doi.org/10.1039/C6SC01167E.

Krist, D.T., Foote, P.K., and Statsyuk, A.V. (2017). UbFluor: A fluorescent thioester to monitor HECT E3 ligase catalysis. Curr. Protoc. Chem. Biol. 9, 11-37. https:// doi.org/10.1002/cpch.17.

Kulathu, Y. (2016). Novel diubiquitin probes expand the chemical toolkit to study DUBs. Cell Chem. Biol. 23, 432-434. https://doi.org/10.1016/j. chembiol.2016.04.001.

Kumar, K.S., Spasser, L., Erlich, L.A., Bavikar, S.N., and Brik, A. (2010). Total chemical synthesis of di-ubiquitin chains. Angew. Chem. Int. Ed. Engl. 49, 9126-9131. https://doi.org/10.1002/anie.201003763.

Kwon, Y.T., and Ciechanover, A. (2017). The ubiquitin code in the ubiquitin-proteasome system and autophagy. Trends Biochem. Sci. 42, 873-886.

Lam, Y.A., Xu, W., DeMartino, G.N., and Cohen, R.E. (1997). Editing of ubiquitin conjugates by an isopeptidase in the $26 \mathrm{~S}$ proteasome. Nature $385,737-$ 740. https://doi.org/10.1038/385737a0.

Lamberto, I., Liu, X., Seo, H.S., Schauer, N.J., Iacob, R.E., Hu, W., Das, D., Mikhailova, T., Weisberg, E.L., Engen, J.R., et al. (2017). Structure-guided development of a potent and selective non-covalent active-site inhibitor of USP7. Cell Chem. Biol. 24, 1490-1500.e11.

Leidecker, O., Matic, I., Mahata, B., Pion, E., and Xirodimas, D.P. (2012). The ubiquitin E1 enzyme Ube1 mediates NEDD8 activation under diverse stress conditions. Cell Cycle 11, 1142-1150. https://doi.org/10.4161/ cc.11.6.19559.

Levin-Kravets, O., Tanner, N., Shohat, N., Attali, I., Keren-Kaplan, T., Shusterman, A., Artzi, S., Varvak, A., Reshef, Y., Shi, X., et al. (2016). A bacterial genetic selection system for ubiquitylation cascade discovery. Nat. Methods 13, 945-952. https://doi.org/10.1038/ nmeth.4003.

Li, G., Liang, Q. Gong, P., Tencer, A.H., and Zhuang, Z. (2014). Activity-based diubiquitin probes for elucidating the linkage specificity of deubiquitinating enzymes. Chem. Commun. 50, 216-218. https://doi. org/10.1039/c3cc47382a.
Liu, Q. Kistemaker, H.A.V., Bhogaraju, S., Dikic, I., Overkleeft, H.S., van der Marel, G.A., Ovaa, H., van der Heden van Noort, G.J., and Filippov, D.V. (2018). A general approach towards triazole-linked adenosine diphosphate ribosylated peptides and proteins. Angew. Chem. Int. Ed. Engl. 57, 1659-1662. https://doi. org/10.1002/anie.201710527.

Love, K.R., Pandya, R.K., Spooner, E., and Ploegh, H.L. (2009). Ubiquitin C-terminal electrophiles are activitybased probes for identification and mechanistic study of ubiquitin conjugating machinery. ACS Chem. Biol. 4, 275-287. https://doi.org/10.1021/cb9000348.

Lu, X., Olsen, S.K., Capili, A.D., Cisar, J.S., Lima, C.D., and Tan, D.S. (2010). Designed semisynthetic protein inhibitors of Ub/Ubl E1 activating enzymes. J. Am. Chem. Soc. 132, 1748-1749. https://doi.org/10.1021/ ja9088549.

Maculins, T., Carter, N., Dorval, T., Hudson, K., Nissink, J.W., Hay, R.T., and Alwan, H. (2016). A generic platform for cellular screening against ubiquitin ligases. Sci. Rep. 6, 18940. https://doi.org/10.1038/srep18940.

Madiraju, C., Welsh, K., Cuddy, M.P., Godoi, P.H., Pass, I., Ngo, T., Vasile, S., Sergienko, E.A., Diaz, P., Matsuzawa, S., et al. (2012). TR-FRET-based highthroughput screening assay for identification of UBC13 inhibitors. J. Biomol. Screen. 17, 163-176. https://doi. org/10.1177/1087057111423417.

Marblestone, J.G., Larocque, J.P., Mattern, M.R., and Leach, C.A. (2012). Analysis of ubiquitin E3 ligase activity using selective polyubiquitin binding proteins. Biochim. Biophys. Acta 1823, 2094-2097. https://doi. org/10.1016/j.bbamcr.2012.06.013.

McCann, A.P., Scott, C.J., Van Schaeybroeck, S., and Burrows, J.F. (2016). Deubiquitylating enzymes in receptor endocytosis and trafficking. Biochem. J. 473, 4507-4525.

McGouran, J.F., Gaertner, S.R., Altun, M., Kramer, H.B., and Kessler, B.M. (2013). Deubiquitinating enzyme specificity for ubiquitin chain topology profiled by di-ubiquitin activity probes. Chem. Biol. 20, 1447-1455. https://doi.org/10.1016/j.chembiol.2013.10.012.

Mevissen, T.E., Hospenthal, M.K., Geurink, P.P., Elliott, P.R., Akutsu, M., Arnaudo, N., Ekkebus, R., Kulathu, Y., Wauer, T., El Oualid, F., et al. (2013). OTU deubiquitinases reveal mechanisms of linkage specificity and enable ubiquitin chain restriction analysis. Cell 154, 169-184. https://doi.org/10.1016/j.cell.2013.05.046.

Mevissen, T.E.T., Kulathu, Y., Mulder, M.P.C., Geurink, P.P., Maslen, S.L., Gersch, M., Elliott, P.R., Burke, J.E., van Tol, B.D.M., Akutsu, M., et al. (2016). Molecular basis of Lys11-polyubiquitin specificity in the deubiquitinase Cezanne. Nature 538, 402-405. https:// doi.org/10.1038/nature19836.

Meyer, H.J., and Rape, M. (2014). Enhanced protein degradation by branched ubiquitin chains. Cell 157, 910-921. https://doi.org/10.1016/j.cell.2014.03.037.

Michel, M.A., Elliott, P.R., Swatek, K.N., Simicek, M., Pruneda, J.N., Wagstaff, J.L., Freund, S.M., and Komander, D. (2015). Assembly and specific recognition of $\mathrm{k} 29$ - and $\mathrm{k} 33$-linked polyubiquitin. Mol. Cell 58, 95-109. https://doi.org/10.1016/j. molcel.2015.01.042. 
Michel, M.A., Swatek, K.N., Hospenthal, M.K., and Komander, D. (2017). Ubiquitin linkage-specific affimers reveal insights into K6-linked ubiquitin signaling. Mol. Cell 68, 233-246.e5.

Mootz, H.D. (2009). Split inteins as versatile tools for protein semisynthesis. Chembiochem 10, 2579-2589. https://doi.org/10.1002/cbic.200900370.

Mulder, M.P.C., El Oualid, F., ter Beek, J., and Ovaa, H. (2014). A native chemical ligation handle that enables the synthesis of advanced activity-based probes: diubiquitin as a case study. Chembiochem 15, 946-949. https://doi.org/10.1002/cbic.201402012.

Mulder, M.P.C., Witting, K., Berlin, I., Pruneda, J.N., Wu, K.P., Chang, J.G., Merkx, R., Bialas, J., Groettrup, M., Vertegaal, A.C., et al. (2016). A cascading activitybased probe sequentially targets E1-E2-E3 ubiquitin enzymes. Nat. Chem. Biol. 12, 523-530. https://doi. org/10.1038/nchembio.2084.

Mulder, M.P.C., Merkx, R., Witting, K.F., Hameed, D.S., El Atmioui, D., Lelieveld, L., Liebelt, F., Neefjes, J., Berlin, I., Vertegaal, A.C.O., et al. (2018). Total chemical synthesis of SUMO and SUMO-based probes for profiling the activity of SUMO-specific proteases. Angew. Chem. Int. Ed. Engl. 57, 8958-8962. https://doi.org/10.1002/ anie.201803483.

Muratani, M., and Tansey, W.P. (2003). How the ubiquitinproteasome system controls transcription. Nat. Rev. Mol. Cell Biol. 4, 192-201. https://doi.org/10.1038/ nrm1049.

Nag, S., Qin, J., Srivenugopal, K.S., Wang, M., and Zhang, R. (2013). The MDM2-p53 pathway revisited. J. Biomed. Res. 27, 254-271. https://doi.org/10.7555/ JBR.27.20130030.

Nie, M., and Boddy, M.N. (2016). Cooperativity of the SUMO and ubiquitin pathways in genome stability. Biomolecules 6, 14. https://doi.org/10.3390/ biom6010014.

Ogunkoya, A.O., Pattabiraman, V.R., and Bode, J.W. (2012). Sequential $\alpha$-ketoacid-hydroxylamine (KAHA) ligations: synthesis of $\mathrm{C}$-terminal variants of the modifier protein UFM1. Angew. Chem. Int. Ed. Engl. 51, 9693-9697. https://doi.org/10.1002/anie.201204144.

Ohtake, F., and Tsuchiya, H. (2017). The emerging complexity of ubiquitin architecture. J. Biochem. 161, 125-133. https://doi.org/10.1093/jb/mvw088.

Ohtake, F., Saeki, Y., Sakamoto, K., Ohtake, K., Nishikawa, H., Tsuchiya, H., Ohta, T., Tanaka, K., and Kanno, J. (2015). Ubiquitin acetylation inhibits polyubiquitin chain elongation. EMBO Rep. 16, 192-201. https://doi. org/10.15252/embr.201439152.

Olsen, S.K., and Lima, C.D. (2013). Structure of a ubiquitin E1-E2 complex: insights to E1-E2 thioester transfer. Mol. Cell 49, 884-896. https://doi.org/10.1016/j. molcel.2013.01.013.

Orcutt, S.J., Wu, J., Eddins, M.J., Leach, C.A., and Strickler, J.E. (2012). Bioluminescence assay platform for selective and sensitive detection of $\mathrm{Ub} / \mathrm{Ubl}$ proteases. Biochim. Biophys. Acta 1823, 2079-2086. https://doi. org/10.1016/j.bbamcr.2012.06.004.

Ovaa, H., Kessler, B.M., Rolén, U., Galardy, P.J., Ploegh, H.L., and Masucci, M.G. (2004). Activity-based ubiquitinspecific protease (USP) profiling of virus-infected and malignant human cells. Proc. Natl. Acad. Sci. U.S.A. 101, 2253-2258.

Pan, M., Gao, S., Zheng, Y., Tan, X., Lan, H., Tan, X., Sun, D., Lu, L., Wang, T., Zheng, Q. et al. (2016). Quasi-racemic $\mathrm{X}$-ray structures of K27-linked ubiquitin chains prepared by total chemical synthesis. J. Am. Chem. Soc. 138, 7429-7435. https://doi.org/10.1021/jacs.6b04031.

Pao, K.C., Stanley, M., Han, C., Lai, Y.C., Murphy, P., Balk, K., Wood, N.T., Corti, O., Corvol, J.C., Muqit, M.M., et al. (2016). Probes of ubiquitin E3 ligases enable systematic dissection of parkin activation. Nat. Chem. Biol. 12, 324-331. https://doi.org/10.1038/nchembio.2045.

Pao, K.C., Wood, N.T., Knebel, A., Rafie, K., Stanley, M., Mabbitt, P.D., Sundaramoorthy, R., Hofmann, K., van Aalten, D.M.F., and Virdee, S. (2018). Activitybased E3 ligase profiling uncovers an E3 ligase with esterification activity. Nature 556, 381-385. https://doi. org/10.1038/s41586-018-0026-1.

Park, S., Krist, D.T., and Statsyuk, A.V. (2015). Protein ubiquitination and formation of polyubiquitin chains without ATP, E1 and E2 enzymes. Chem. Sci. 6, 17701779. https://doi.org/10.1039/c4sc02340d.

Pickart, C.M., and Rose, I.A. (1986). Mechanism of ubiquitin carboxyl-terminal hydrolase. Borohydride and hydroxylamine inactivate in the presence of ubiquitin. J. Biol. Chem. 261, 10210-10217.

Pruneda, J.N., Durkin, C.H., Geurink, P.P., Ovaa, H., Santhanam, B., Holden, D.W., and Komander, D. (2016). The molecular basis for ubiquitin and ubiquitinlike specificities in bacterial effector proteases. Mol. Cell 63, 261-276.

Puvar, K., Zhou, Y., Qiu, J., Luo, Z.Q., Wirth, M.J., and Das, C. (2017). Ubiquitin chains modified by the bacterial ligase SdeA are protected from deubiquitinase hydrolysis. Biochemistry 56, 4762-4766. https://doi. org/10.1021/acs.biochem.7b00664.

Qu, Q. Pan, M., Gao, S., Zheng, Q.Y., Yu, Y.Y., Su, J.C., Li, X., and Hu, H.G. (2018). A highly efficient synthesis of polyubiquitin chains. Adv. Sci. 5, 1800234. https://doi. org/10.1002/advs.201800234.

Ramage, R., Green, J., Muir, T.W., Ogunjobi, O.M., Love, S., and Shaw, K. (1994). Synthetic, structural and biological studies of the ubiquitin system: the total chemical synthesis of ubiquitin. Biochem. J. 299, 151-158.

Regnström, K., Yan, J., Nguyen, L., Callaway, K., Yang, Y., Diep, L., Xing, W., Adhikari, A., Beroza, P., Hom, R.K., et al. (2013). Label free fragment screening using surface plasmon resonance as a tool for fragment finding - analyzing parkin, a difficult CNS target. PLOS ONE 8, e66879. https://doi.org/10.1371/journal. pone. 0066879 .

Reverdy, C., Conrath, S., Lopez, R., Planquette, C., Atmanene, C., Collura, V., Harpon, J., Battaglia, V., Vivat, V., Sippl, W., et al. (2012). Discovery of specific inhibitors of human USP7/HAUSP deubiquitinating enzyme. Chem. Biol. 19, 467-477. https://doi.org/10.1016/j. chembiol.2012.02.007.

Rösner, D., Schneider, T., Schneider, D., Scheffner, M., and Marx, A. (2015). Click chemistry for targeted protein ubiquitylation and ubiquitin chain formation. Nat. Protoc. 10, 1594-1611. https://doi.org/10.1038/ nprot.2015.106. 
Saghatelian, A., Jessani, N., Joseph, A., Humphrey, M., and Cravatt, B.F. (2004). Activity-based probes for the proteomic profiling of metalloproteases. Proc. Natl. Acad. Sci. U.S.A. 101, 10000-10005. https://doi. org/10.1073/pnas.0402784101.

Scheffner, M., and Kumar, S. (2014). Mammalian HECT ubiquitin-protein ligases: biological and pathophysiological aspects. Biochim. Biophys. Acta 1843, 61-74. https://doi.org/10.1016/j. bbamcr.2013.03.024.

Shanmugham, A., Fish, A., Luna-Vargas, M.P., Faesen, A.C., El Oualid, F., Sixma, T.K., and Ovaa, H. (2010). Nonhydrolyzable ubiquitin-isopeptide isosteres as deubiquitinating enzyme probes. J. Am. Chem. Soc. 132, 8834-8835. https://doi.org/10.1021/ja101803s.

Sheng, Y., Saridakis, V., Sarkari, F., Duan, S., Wu, T., Arrowsmith, C.H., and Frappier, L. (2006). Molecular recognition of p53 and MDM2 by USP7/HAUSP. Nat. Struct. Mol. Biol. 13, 285-291.

Singh, R.K., Sundar, A., and Fushman, D. (2014). Nonenzymatic rubylation and ubiquitination of proteins for structural and functional studies. Angew. Chem. Int. Ed. Engl. 53, 6120-6125. https://doi.org/10.1002/ anie. 201402642 .

Sowa, M.E., Bennett, E.J., Gygi, S.P., and Harper, J.W. (2009). Defining the human deubiquitinating enzyme interaction landscape. Cell 138, 389-403. https://doi. org/10.1016/j.cell.2009.04.042.

Spratt, D.E., Walden, H., and Shaw, G.S. (2014). RBR E3 ubiquitin ligases: new structures, new insights, new questions. Biochem. J. 458, 421-437. https://doi. org/10.1042/BJ20140006.

Stanley, M., Han, C., Knebel, A., Murphy, P., Shpiro, N., and Virdee, S. (2015). Orthogonal thiol functionalization at a single atomic center for profiling transthiolation activity of E1 activating enzymes. ACS Chem. Biol. 10, 15421554. https://doi.org/10.1021/acschembio.5b00118.

Tang, S., Liang, L.J., Si, Y.Y., Gao, S., Wang, J.X., Liang, J., Mei, Z., Zheng, J.S., and Liu, L. (2017). Practical chemical synthesis of atypical ubiquitin chains by using an isopeptide-linked Ub isomer. Angew. Chem. Int. Ed. Engl. 56, 13333-13337. https://doi.org/10.1002/ anie.201708067.

Tirat, A., Schilb, A., Riou, V., Leder, L., Gerhartz, B., Zimmermann, J., Worpenberg, S., Eidhoff, U., Freuler, F., Stettler, T., et al. (2005). Synthesis and characterization of fluorescent ubiquitin derivatives as highly sensitive substrates for the deubiquitinating enzymes UCH-L3 and USP-2. Anal. Biochem. 343, 244-255.

Trang, V.H., Valkevich, E.M., Minami, S., Chen, Y.C., Ge, Y., and Strieter, E.R. (2012). Nonenzymatic polymerization of ubiquitin: single-step synthesis and isolation of discrete ubiquitin oligomers. Angew. Chem. Int. Ed. Engl. 51, 13085-13088. https://doi.org/10.1002/ anie.201207171.

Uchida, C., and Kitagawa, M. (2016). RING-, HECT-, and RBR-type E3 ubiquitin ligases: involvement in human cancer. Curr. Cancer Drug Targets 16, 157-174.

Valkevich, E.M., Guenette, R.G., Sanchez, N.A., Chen, Y.C., Ge, Y., and Strieter, E.R. (2012). Forging isopeptide bonds using thiol-ene chemistry: site-specific coupling of ubiquitin molecules for studying the activity of isopeptidases. J. Am. Chem. Soc. 134, 6916-6919. https://doi.org/10.1021/ja300500a.

van der Heden van Noort, G.J., Kooij, R., Elliott, P.R., Komander, D., and Ovaa, H. (2017). Synthesis of poly-ubiquitin chains using a bifunctional ubiquitin monomer. Org. Lett. 19, 6490-6493. https://doi. org/10.1021/acs.orglett.7b03085.

van der Heden van Noort, G., Talavera Ormeno, C., Van Dalen, D., and Ovaa, H. (2018). One-step chemical synthesis of native Met1-linked poly-ubiquitin chains. ChemBioChem 20, 62-65. https://doi.org/10.1002/ cbic.201800520.

van Tilburg, G.B., Elhebieshy, A.F., and Ovaa, H. (2016). Synthetic and semi-synthetic strategies to study ubiquitin signaling. Curr. Opin. Struct. Biol. 38, 92-101. https://doi.org/10.1016/j.sbi.2016.05.022.

Vittal, V., Stewart, M.D., Brzovic, P.S., and Klevit, R.E. (2015). Regulating the regulators: recent revelations in the control of E3 ubiquitin ligases. J. Biol. Chem. 290, 21244-21251. https://doi.org/10.1074/jbc. R115.675165.

Walden, H., and Rittinger, K. (2018). RBR ligase-mediated ubiquitin transfer: a tale with many twists and turns. Nat. Struct. Mol. Biol. 25, 440-445. https://doi. org/10.1038/s41594-018-0063-3.

Wals, K., and Ovaa, H. (2014). Unnatural amino acid incorporation in E. coli: current and future applications in the design of therapeutic proteins. Front. Chem. 2, 15. https://doi.org/10.3389/fchem.2014.00015.

Wang, D., Ma, L., Wang, B., Liu, J., and Wei, W. (2017). E3 ubiquitin ligases in cancer and implications for therapies. Cancer Metastasis Rev. 36, 683-702. https:// doi.org/10.1007/s10555-017-9703-z.

Wang, X.A., Kurra, Y., Huang, Y., Lee, Y.J., and Liu, W.R. (2014). E1-catalyzed ubiquitin C-terminal amidation for the facile synthesis of deubiquitinase substrates. Chembiochem 15, 37-41. https://doi.org/10.1002/ cbic. 201300608 .

Weber, A., Elliott, P.R., Pinto-Fernandez, A., Bonham, S., Kessler, B.M., Komander, D., El Oualid, F., and Krappmann, D. (2017). A linear diubiquitin-based probe for efficient and selective detection of the deubiquitinating enzyme OTULIN. Cell Chem. Biol. 24, 1299-1313.e7.

Weller, C.E., Huang, W., and Chatterjee, C. (2014). Facile synthesis of native and protease-resistant ubiquitylated peptides. Chembiochem 15, 1263-1267. https://doi. org/10.1002/cbic.201402135.

Wiener, R., Zhang, X., Wang, T., and Wolberger, C. (2012). The mechanism of OTUB1-mediated inhibition of ubiquitination. Nature 483, 618-622. https://doi. org/10.1038/nature 10911 .

Witting, K.F., van der Heden van Noort, G.J., Kofoed, C., Talavera Ormeño, C., El Atmioui, D., Mulder, M.P.C., and Ovaa, H. (2018). Generation of the UFM1 toolkit for profiling UFM1-specific proteases and ligases. Angew. Chem. Int. Ed. Engl. 57, 14164-14168. https:// doi.org/10.1002/anie.201809232.

Wucherpfennig, T.G., Pattabiraman, V.R., Limberg, F.R., Ruiz-Rodríguez, J., and Bode, J.W. (2014). Traceless preparation of C-terminal $\alpha$-ketoacids for chemical protein synthesis by $\alpha$-ketoacid-hydroxylamine ligation: synthesis of SUMO2/3. Angew. Chem. Int. 
Ed. Engl. 53, 12248-12252. https://doi.org/10.1002/ anie.201407014.

Xin, B.T., van Tol, B.D.M., Ovaa, H., and Geurink, P.P. (2018). Native chemical ligation at methionine bioisostere norleucine allows for $\mathrm{N}$-terminal chemical protein ligation. Org. Biomol. Chem. 16, 6306-6315. https://doi.org/10.1039/c8ob01627e.

Xu, P., Duong, D.M., Seyfried, N.T., Cheng, D., Xie, Y., Robert, J., Rush, J., Hochstrasser, M., Finley, D., and Peng, J. (2009). Quantitative proteomics reveals the function of unconventional ubiquitin chains in proteasomal degradation. Cell 137, 133-145. https:// doi.org/10.1016/j.cell.2009.01.041.

Yang, C.S., Jividen, K., Spencer, A., Dworak, N., Ni, L., Oostdyk, L.T., Chatterjee, M., Kuśmider, B., Reon, B., Parlak, M., et al. (2017). Ubiquitin modification by the E3 ligase/ADP-ribosyltransferase Dtx3L/Parp9. Mol. Cell 66, 503-516.e5.

Yang, R., Pasunooti, K.K., Li, F., Liu, X.W., and Liu, C.F. (2009). Dual native chemical ligation at lysine. J. Am. Chem. Soc. 131, 13592-13593. https://doi. org/10.1021/ja905491p.
Yuan, W.C., Lee, Y.R., Lin, S.Y., Chang, L.Y., Tan, Y.P., Hung, C.C., Kuo, J.C., Liu, C.H., Lin, M.Y., Xu, M., et al. (2014). K33-linked polyubiquitination of coronin 7 by Cul3KLHL20 ubiquitin e 3 ligase regulates protein trafficking. Mol. Cell 54, 586-600. https://doi.org/10.1016/j. molcel.2014.03.035.

Zhang, W., Wu, K.P., Sartori, M.A., Kamadurai, H.B., Ordureau, A., Jiang, C., Mercredi, P.Y., Murchie, R., Hu, J., Persaud, A., et al. (2016). System-wide modulation of HECT E3 ligases with selective ubiquitin variant probes. Mol. Cell 62, 121-136. https://doi.org/10.1016/j. molcel.2016.02.005.

Zhang, X., Smits, A.H., van Tilburg, G.B., Jansen, P.W., Makowski, M.M., Ovaa, H., and Vermeulen, M. (2017). An interaction landscape of ubiquitin signaling. Mol. Cell 65, 941-955.e8.

Zhao, B., Bhuripanyo, K., Schneider, J., Zhang, K., Schindelin, H., Boone, D., and Yin, J. (2012). Specificity of the E1-E2-E3 enzymatic cascade for ubiquitin $\mathrm{C}$-terminal sequences identified by phage display. ACS Chem. Biol. 7, 2027-2035. https://doi.org/10.1021/ cb300339p. 